\title{
Removing Infragravity-Wave-Induced Noise from Ocean-Bottom Seismographs (OBS) Data Deployed Offshore of Taiwan
}

\author{
by Ban-Yuan Kuo, Spahr C. Webb, Ching-Ren Lin, Wen-Tzong Liang, and Nai-Chi Hsiao
}

\begin{abstract}
Vertical ocean-bottom seismograph (OBS) data at frequencies below $0.05 \mathrm{~Hz}$ are contaminated by noise induced by infragravity waves. We constructed the transfer function between pressure and velocity data from OBSs deployed in Taiwan waters to remove the wave pressure-induced noise from seismic recordings. Data were analyzed from five portable broadband OBSs deployed each for 10 months at water depths from 1740 to $4600 \mathrm{~m}$ and from a cabled, shallow-buried seismograph (EOS1) installed on the seafloor at $300 \mathrm{~m}$ depth. Removing long-period noise from these OBS data improves the identification of teleseismic phases such as $P, S, S S, P_{\text {diff }}$, and $P K I K P$ that are otherwise ambiguous or unidentifiable. For EOS1, infragravitywave signals completely mask the $P$ and $S$ waveforms in the $10-50$ s period band suitable for centroid moment tensor (CMT) solutions for most of the local events. Application of the transfer functions to predict and remove wave deformation yielded clean prominent $P$ and $S$ waveforms at these periods and aided in the CMT determination for small events jointly with land stations. The relative amplitudes of the wavenumber-normalized transfer function for some of the OBSs are mostly determined by the thickness of the sediment at the OBS site.
\end{abstract}

\section{Introduction}

Portable broadband ocean-bottom seismographs (OBSs) were first deployed in 2006 in the waters east and south of Taiwan (e.g., Lin et al., 2010) to expand the aperture of the island-based seismic array in the initial phase of a long-term ocean-bottom observation project investigating the oblique collision/subduction in the Taiwan region. Because the seafloor deployments were strategically planned to best augment the land stations, the limited amount of data from these sites provides critical constraints on models of the evolution and dynamics in this corner of the Philippine Sea plate (Kuo et al., 2009; Ko et al., 2012; Kuo et al., 2012). In late 2011, a cabled, permanent seafloor observatory was installed offshore northeast Taiwan by the Central Weather Bureau (CWB) of Taiwan for hazard monitoring and mitigation purposes. All OBSs are equipped with a pressure sensor in addition to the three-component seismometer. This new data set opens a new opportunity to advance earthquake monitoring and tsunami warning and to improve our knowledge of the Earth's structure in the Taiwan region.

Ocean waves generate most of the noise recorded by seismometers, and different wave types dominate at different frequencies (Webb, 1998). For frequencies $<0.05 \mathrm{~Hz}$, seismic noise is predominately caused by infragravity waves, which are low-frequency ocean waves derived from interactions of short-period ocean waves refracting and trapped over continental shelves (Webb et al., 1991; Webb and Craw- ford, 1999). The water pressure fluctuations associated with infragravity waves force the seafloor to respond coherently. This bottom motion is registered in the vertical recording of each OBS and can obscure seismic signals in this frequency band. The infragravity-wave signals appear as a hump between 0.01 and $0.05 \mathrm{~Hz}$ in the power spectrum for the OBS vertical component (e.g., Dolenc et al., 2005, 2008; Lin et al., 2010). Because ocean wave amplitudes decay exponentially with depth, the hump is usually conspicuous on spectra only from OBSs at depths less than $2000 \mathrm{~m}$ (e.g., Dolenc et al., 2005, 2008; Lin et al., 2010).

This bottom motion forced by ocean water pressure fluctuations is noise for seismological research that employs seismic phases, but because the motion also reflects how the seafloor responds elastically to loading, it also provides a useable signal: the compliance function. This function depends on the sediment and crustal structure beneath each OBS site (Crawford et al., 1991, 1999). For its noise role, techniques have been developed and used to predict, and thus remove it from the vertical seismic data (e.g., Crawford and Webb, 2000; Dolenc et al., 2007). In this study, we apply the frequency-domain transfer function method (Crawford and Webb, 2000) to data from both portable and permanent OBSs offshore Taiwan to clean the vertical seismograms and enhance the quality of OBS vertical data. We also explored the signal role of the transfer function in a relative sense among 


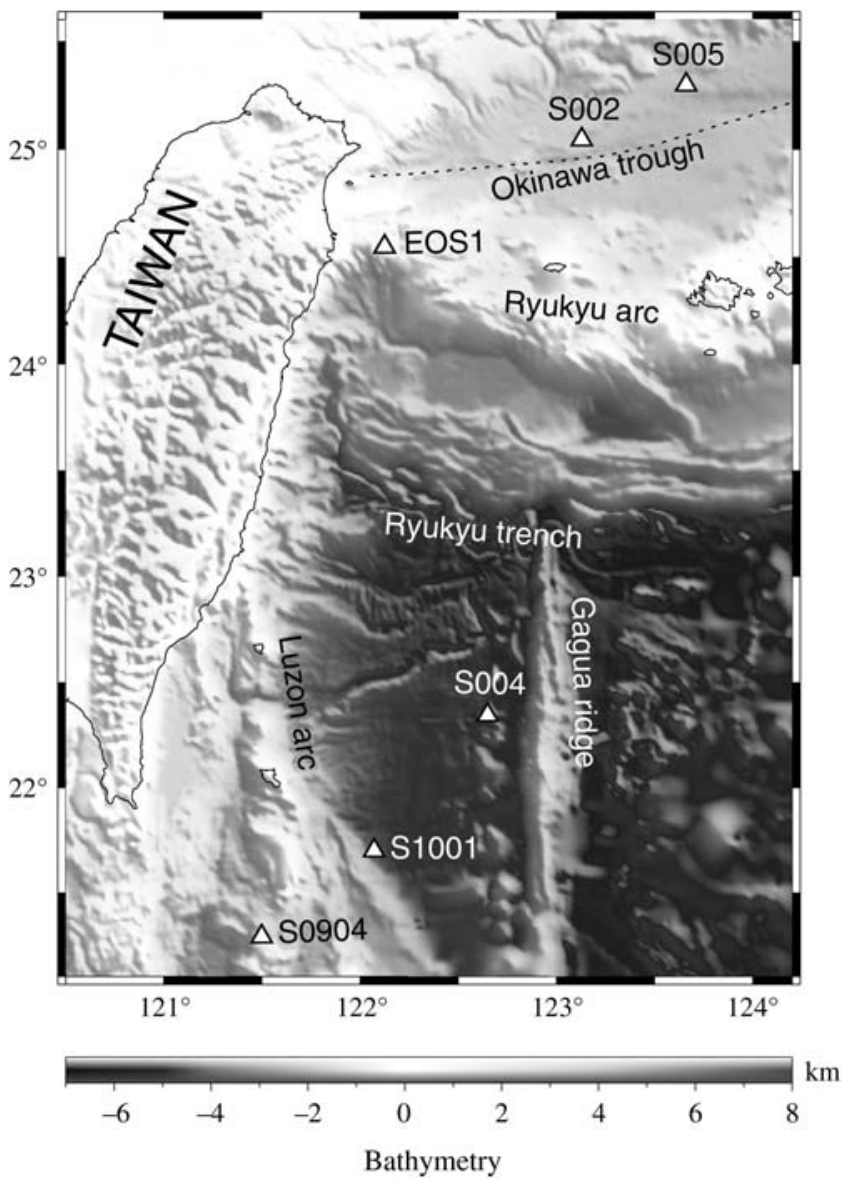

Figure 1. Locations of broadband ocean-bottom seismographs (OBSs) deployed in the Taiwan collision subduction zone since 2006. S002, S004, S005, S0904, and S1001 are portable OBSs. EOS1 hosts a cabled, shallow-buried seafloor observatory. All are equipped with pressure gauges that allow removal of oceanwave-induced noise from seismic data. The water depths for the five portable OBSs are $1740 \mathrm{~m}$ (S002), $4730 \mathrm{~m}$ (S004), $1900 \mathrm{~m}$ (S005), $1230 \mathrm{~m}$ (S0904), and $4670 \mathrm{~m}$ (S1001). EOS1 is buried in the sediment at a water depth of $300 \mathrm{~m}$

different OBSs. This study serves as a first step toward a comprehensive utilization of all channels of OBS data collected in the Taiwan region.
Data

OBSs S002 and S004 were deployed in the Okinawa trough and the Huatung basin in 2006-2007 at water depths of 1740 and $4730 \mathrm{~m}$, respectively (Fig. 1). OBS S005 was deployed further east in the Okinawa trough on the $1900 \mathrm{~m}$ deep seafloor in 2007. S0904 and S1001 are OBS sites offshore of southern Taiwan and were deployed in water depths of 1230 and $4670 \mathrm{~m}$, in 2009 and 2010, respectively (see Table 1 for a summary). Each OBS was operational for one campaign period of about 10 months. All were equipped with a broadband (50 Hz-120 s) Güralp CMG-3TC sensor with a sampling rate of $100 \mathrm{~Hz}$ (Lin et al., 2010) and a differential pressure gauge (DPG) with a sampling rate of $20 \mathrm{~Hz}$ (Cox et al., 1984). The DPG response is flat in pressure at short periods, and roughly proportional to the first derivative of pressure at periods from 30 to about $300 \mathrm{~s}$. The response is proportional to the second derivative of pressure at very long period. However, the magnitude of this response has not been rigorously calibrated and the pressure data as recorded were used in constructing the transfer function.

The CWB cabled permanent station EOS1 was installed $45 \mathrm{~km}$ offshore northeast Taiwan on the western extension of the Ryukyu arc at a water depth of $300 \mathrm{~m}$ (Fig. 1). The instrument platform houses a Güralp CMG-3TC extended broadband sensor with a flat velocity response to $360 \mathrm{~s}$ and it was shallowly buried under the surface into the sediment. EOS1 employs a quartz pressure gauge to record absolute pressure with a flat response to at least $300 \mathrm{~s}$. We analyzed 15 months of EOS1 data beginning in December 2011.

Power spectra were calculated on data from S0904, S1001, and EOS1 (Fig. 2) following the procedures in McNamara and Buland (2004). See Lin et al. (2010) for the power spectrum density (PSD) for S002, S004, and S005. The PSDs show a marked noise hump peaking at different frequencies corresponding to infragravity waves. With increasing water depths, the hump and the noise notch shift toward lower frequencies from EOS1 at $300 \mathrm{~m}$ to S0904 at $1230 \mathrm{~m}$ and to $\mathrm{S} 1001$ at $4670 \mathrm{~m}$. The noise level of the infragravity wave also decreases with increasing water depth. The S0904 records were interrupted by instrument glitches,

Table 1

Ocean-Bottom Seismograph (OBS) Site and Data Analysis Parameters

\begin{tabular}{llrcrcc}
\hline OBS & \multicolumn{1}{c}{ Region } & $H^{*}$ & Deployment Period & \multicolumn{1}{c}{$n^{\dagger}$} & $h^{\ddagger}$ & ID $^{\S}$ \\
\hline S002 & Okinawa trough & 1740 & $2006 / 09-2007 / 07$ & 166 & 700 & OBS30 \\
S004 & Huatung basin & 4730 & $2006 / 09-2007 / 07$ & 159 & 486 & OBS33 \\
S005 & Okinawa trough & 1900 & $2007 / 09-2008 / 07$ & 1100 & 567 & OBS30 \\
S0904 & North Luzon trough & 1230 & $2009 / 09-2010 / 07$ & 63 & 276 & OBS33 \\
S1001 & Huatung basin & 4670 & $2010 / 09-2011 / 07$ & 799 & 935 & OBS33 \\
EOS1 & Ryukyu arc & 300 & $2011 / 11$ & 2000 & 900 &
\end{tabular}

\footnotetext{
$* H$, water depth in meter.

$\downarrow$, total number of $3600 \mathrm{~s}$ data window used to construct transfer function.

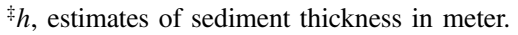

${ }^{\S}$ OBS30 and OBS33 are instrument identification codes. The same code indicates the same pair of seismometer and DPG.
} 


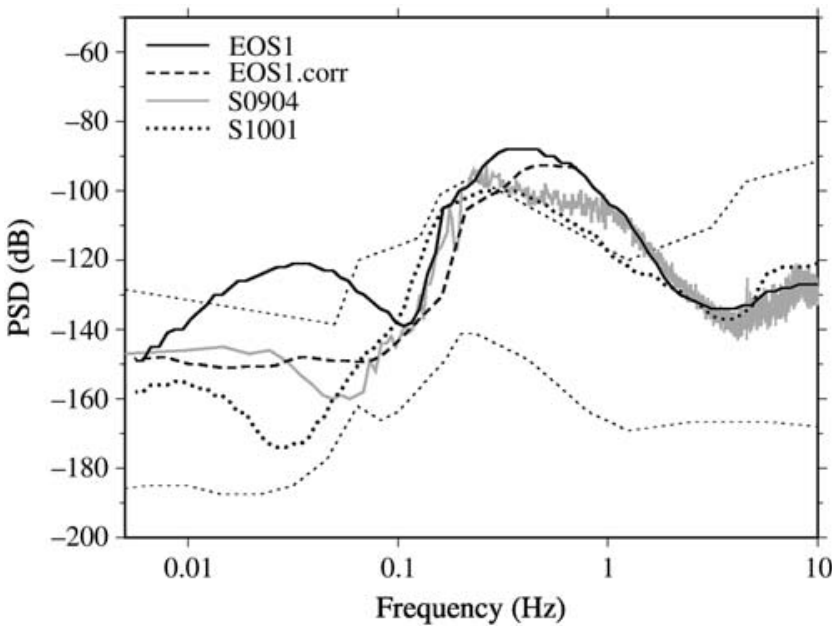

Figure 2. Power spectral density (mode) calculated from vertical data of EOS1 (solid line), S0904 (gray), and S1001 (dotted). See Lin et al. (2010) for S002, S004, and S005. The decreasing amplitude of the noise hump and the decreasing frequency of noise notch with increasing water depth from EOS1 at $300 \mathrm{~m}$ to S0904 at $1230 \mathrm{~m}$ and to $\mathrm{S} 1001$ at $4670 \mathrm{~m}$. Removal of this noise at EOS1 reduces the noise level by a maximum of 25-30 dB (dashed line). The high- and low-noise models of Peterson (1993) (thin dotted line) are shown as references.

and available data were broken into short segments, which cannot be processed using the McNamara and Buland (2004) routine. For S0904, we used the routine "sacpsd" in the "Computer programs in seismology" package maintained in the Saint Louis University Earthquake Center. We also calculated the PSD using "sacpsd" for S1001 and EOS1 and found that the trends in PSD remain the same as that calculated with McNamara and Buland (2004).

Figure 3 shows examples of the time-domain correlation between pressure and vertical displacement. For illustration purposes, we use true displacement with the instrument response removed from the velocity data. To compare with the displacement, DPG data from portable OBSs and the quartz pressure gauge data from EOS1 were also corrected for the instrument response to remove the phase effects. In Figure 3, the vertical displacements correlate almost wiggle by wiggle with the pressure perturbations in the absence of seismic signals. In this figure, DPG data are plotted with pressure positive down, so the vertical displacement of the seafloor is in phase with the seafloor pressure signal, as expected for infragravity-wave loading deformation.

\section{Transfer Function}

To construct the transfer function, it is not necessary to correct for instrument response, and velocity and pressure recordings in units of counts are used in the analysis shown next. True displacement can be obtained by deconvolving the instrument response after the noise-removal process is completed on the velocity data. The coherence in the frequency domain is calculated following Crawford and Webb (2000) (a)

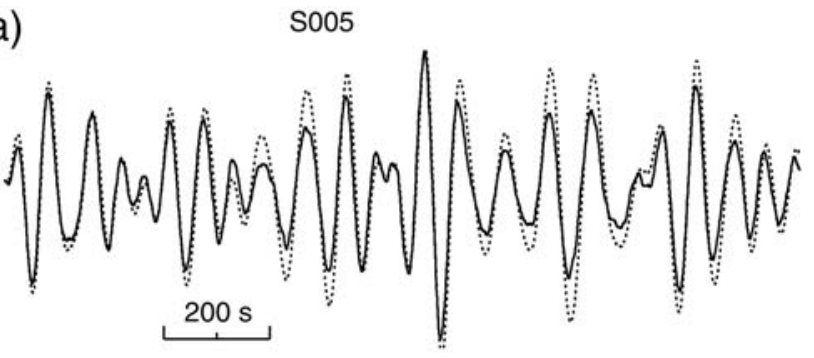

(b)

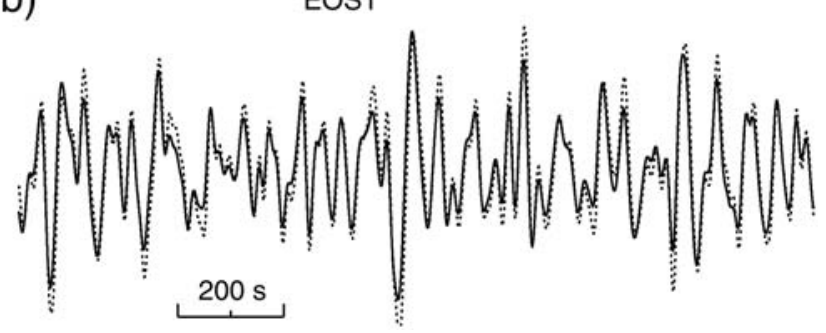

Figure 3. Example of time-domain correlation between pressure gauge data (dotted line) and vertical displacement (solid line) in a $1500 \mathrm{~s}$ time window for (a) S005 on Julian day 26 of 2008 and (b) EOS1 on Julian day 42 of 2012, for $0.01-0.1 \mathrm{~Hz}$ and $0.005-$ $0.1 \mathrm{~Hz}$ frequency band, respectively. The instrument responses were deconvolved from pressure gauge and seismic data for the purpose of comparison. Scales are arbitrarily adjusted for the clarity of phase match between the two traces. Positive pressure perturbation is down. EOS1 at $300 \mathrm{~m}$ water depth has more high-frequency noise than S005 at $1900 \mathrm{~m}$ water depth.

$$
\gamma_{\mathrm{vp}}(f)=\frac{G_{\mathrm{vp}}(f)}{\sqrt{G_{\mathrm{vv}}(f) \times G_{\mathrm{pp}}(f)}} .
$$

Here, the autospectra for vertical velocity $G_{\mathrm{vv}}$ and pressure $G_{\mathrm{pp}}$, and the cross spectrum between the two $G_{\mathrm{vp}}$ are defined as

$$
\begin{gathered}
G_{\mathrm{vv}}(f)=\frac{1}{n \times T} \sum_{i=1}^{n}\left|V_{i}(f)\right|^{2} \\
G_{\mathrm{pp}}(f)=\frac{1}{n \times T} \sum_{i=1}^{n}\left|P_{i}(f)\right|^{2} \\
G_{\mathrm{vp}}(f)=\frac{1}{n \times T} \sum_{i=1}^{n} V_{i}^{*}(f) P_{i}(f),
\end{gathered}
$$

in which $V_{i}(f)$ and $P_{i}(f)$ are the Fourier-transformed velocity and pressure at frequency $f$ for the $i$ th data window, $V_{i}^{*}$ is the conjugate of $V_{i}, n$ is the number of data windows, and $T=3600 \mathrm{~s}$ the length of each window. In this operation, both velocity and pressure were downsampled from 0.01 to $0.1 \mathrm{~s}$ after an antialiasing filter was applied to the original data. The mean of each data window was removed, and the data tapered using a 5\% cosine bell at each end. This data window was then padded with zeros to extend to the nearest power of 2 points for the spectral estimation. 
We chose data with little earthquake energy and avoided noise from instrument glitches by first calculating the coherence for each one-hour data window to sort out those with high coherence over a wide frequency range to be used in the ensemble calculation in equations (2)-(4). The coherence for each one-hour data window was calculated by dividing the data window into four subwindows, that is, $n=4$ and $T=900 \mathrm{~s}$ in equations (2)-(4), The result has been checked against the two-hour coherence with four subwindows and $T=1800 \mathrm{~s}$ and proven to be effective in distinguishing useful data. Although large-amplitude Rayleigh waves and body waves from local events may yield high coherence between pressure and velocity, the coherence may present a narrow hump, rather than a plateau, or approach one at low frequencies (e.g., for portable OBSs), and these data windows would be checked and eliminated in this step. We then visually inspected the remaining data to remove those suspected to still be significantly polluted by seismic waves. This second step eliminated only a very small portion of the total data set of the study. For EOS1, the infragravity-wave energy is so strong and instruments so stable that coherence estimates based on one day of data (24 data windows) are sufficient. With carefully selected data windows, the coherence stabilizes when $n>40$ for the portable OBSs S002, S004, S005, S0904, and S1001 (several days of data), and within one day for EOS1 (Fig. 4). Sorting the data through coherence estimates based on one-hour data windows makes it possible to extract the coherence and the transfer function from the interrupted records of S0904.

The coherence for EOS1 is almost unity to frequencies of at least $0.06 \mathrm{~Hz}$ because the water is sufficiently shallow that the pressure signal from infragravity waves reaches the seafloor at these short periods. The infragravity-wave spectrum in shallow water is also higher than at deeper sites. For other OBSs, the coherence displays a much narrower plateau than that for EOS1 with the peak shifting toward lower frequencies with increasing water depth (Fig. 4). The phase of the coherence stems from the phase difference between pressure and velocity uncorrected for instrument responses.

Following Crawford and Webb (2000), the transfer function is defined as

$$
T_{\mathrm{vp}}(f)=\gamma_{\mathrm{vp}}(f) \times \sqrt{\frac{G_{\mathrm{vv}}(f)}{G_{\mathrm{pp}}(f)}}
$$

Figure 5a-g shows the amplitudes of transfer functions for all OBSs. The phase of the transfer function is the same as the phase of the coherence. The transfer functions are significant only in frequency bands for which the coherence is significantly above zero. The transfer functions increase smoothly with frequency before falling as the high-frequency limit of the infragravity band is approached, at which the coherence between pressure and displacements disappears. For EOS1, it is stable up to at least $0.06 \mathrm{~Hz}$ reflecting the high coherence seen across this frequency range.
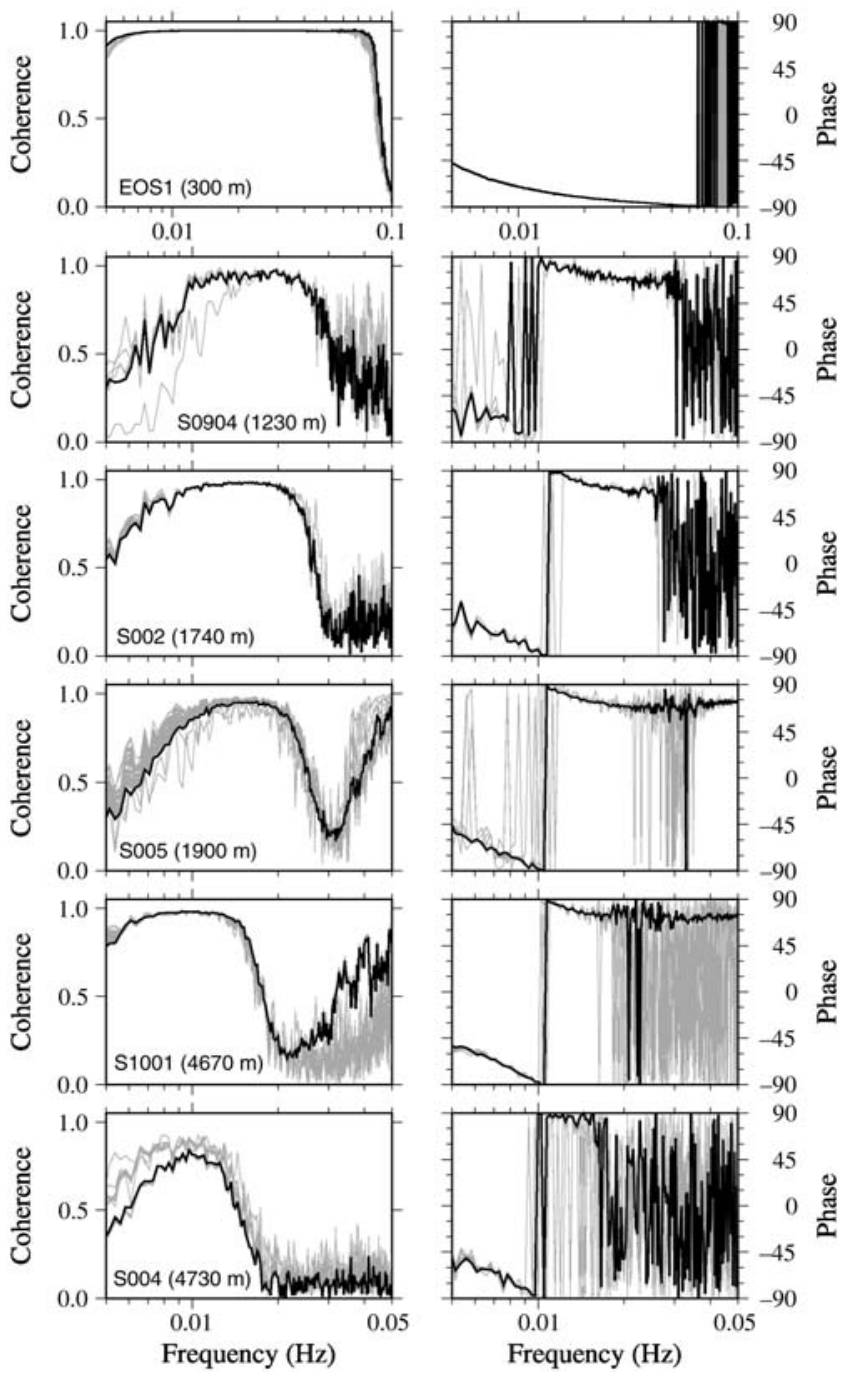

Figure 4. Coherence between uncorrected velocity and pressure data, with amplitude on the left column and phase on the right column, for each OBS the name for which is annotated on the amplitude panel. The frequency range on the plot is $0.005-0.1 \mathrm{~Hz}$ for EOS1 and $0.005-0.05 \mathrm{~Hz}$ for all other OBSs. For all OBSs but EOS1, each gray line is the coherence for stacks of one-hour data windows up to $n=20,30$, and so on in equations (2)-(4). For EOS1, the gray line is the coherence for stacks of one-day records, starting at the 20th day incrementing with 10 days. These gray lines converge to the solid line, the final coherence. From top to bottom the OBSs are arranged with increasing water depth (in parenthesis). The generally decreasing maximum frequency for the highcoherence plateau with water depth. The phase around $90^{\circ}$ represents the phase between velocity and pressure and the phase of both instruments.

\section{Removal of Noise}

The pressure effect is removed from the velocity data in the frequency domain by subtracting from the Fourier transform of a one-hour long vertical component record a prediction for the wave-loading noise. This prediction is estimated as the product of Fourier transform of the pressure record from the same one-hour interval multiplied by the conjugate of the transfer function for the site, 

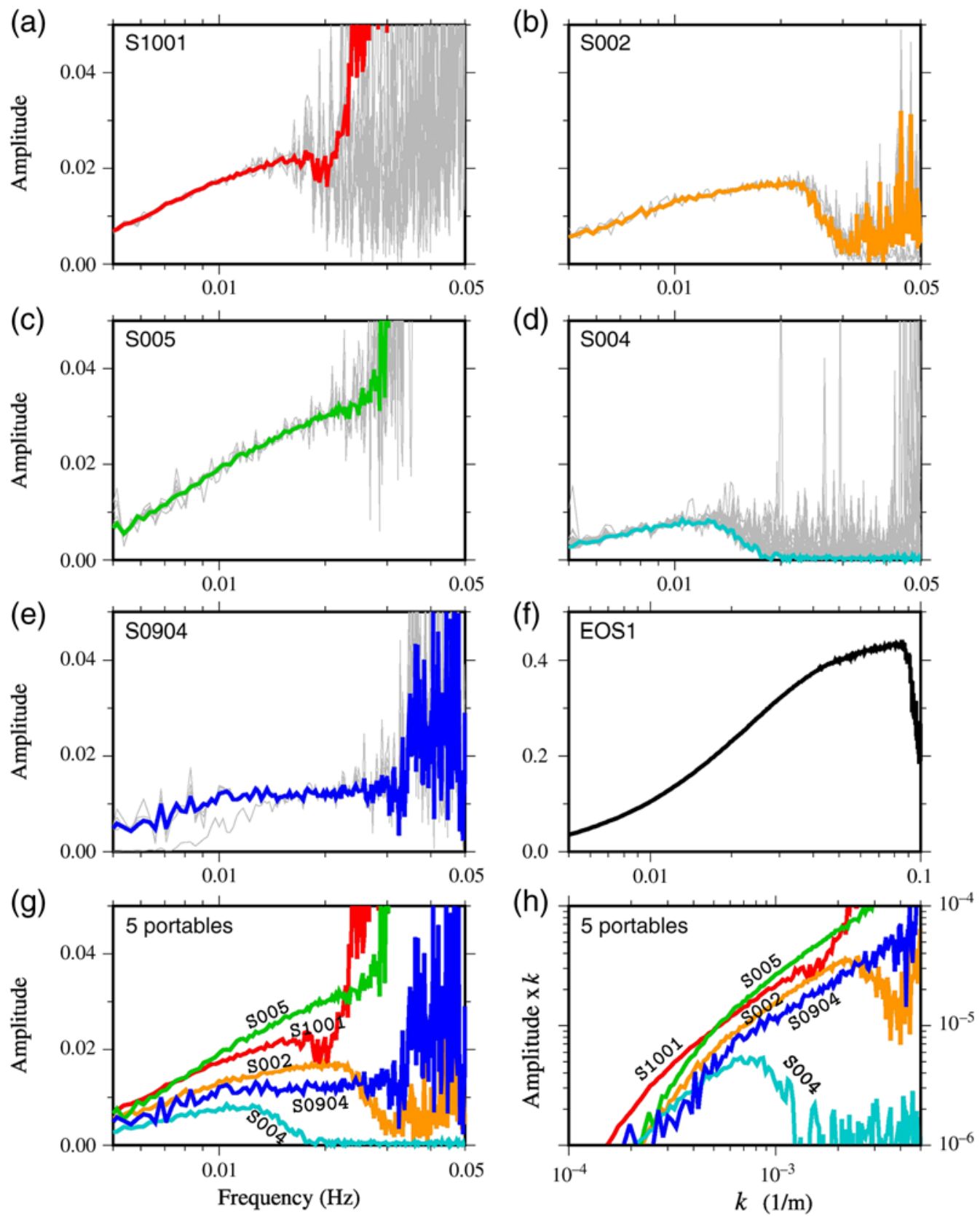

Figure 5. Amplitude of transfer functions for all the OBSs. The frequency range is $0.005-0.1 \mathrm{~Hz}$ for EOS 1 and $0.005-0.05 \mathrm{~Hz}$ for other OBSs. From (a) to (f) the OBSs are ordered with decreasing estimates of sediment thickness (Table 1). The lighter gray lines represent accumulative transfer functions calculated the same way as the coherence in Figure 4. EOS1 shows a rapid convergence toward the final transfer function and the gray lines are almost invisible. (g) The comparison among portable OBSs, and (h) the same comparison but for wavenumber-normalized transfer function versus $k$, which partially neutralizes the effect of water depth. In (h), the relative amplitudes between S0904 and S1001 may be dominated by the significant difference (276 versus $935 \mathrm{~m}$ ) in sediment thickness (see Discussion section). EOS1 has different instrument specs and is not compared with portable OBSs. The color version of this figure is available only in the electronic edition.

$$
V^{\prime}(f)=V(f)-T_{\mathrm{vp}}^{*} \times P(f),
$$

in which $T_{\mathrm{vp}}^{*}$ is the conjugate of $T_{\mathrm{vp}}$ and $V^{\prime}(f)$ represents the cleaned velocity data. An inverse Fourier transform is then applied to $V^{\prime}(f)$ to obtain the cleaned record in the time domain. Examples of the application of equation (6) for teleseismic events on S005 and EOS1 are shown in Figure 6. In these examples, the seismic phases are sufficiently away from the beginning of the $3600 \mathrm{~s}$ data window. The $P, S$, and SS arrivals are ambiguous in the original S005 displacement seismograms for a teleseismic event but are evident after removing the infragravity-wave-induced long-period noise. At EOS1, the $P_{\text {diff }}$ and PKIKP waveforms are distorted or invisible but revealed clearly on the wave-noise-suppressed 
(a)

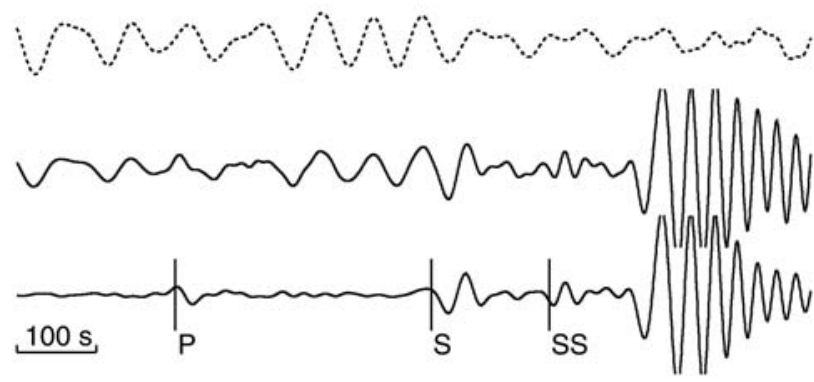

(b)

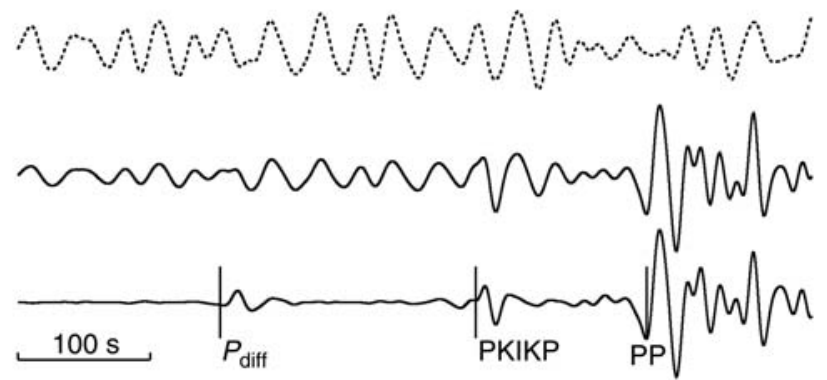

Figure 6. Removal of noise by using the transfer function. In each panel, the three traces from top down are pressure (dotted line), vertical displacement, and vertical displacement cleaned with transfer function. Pressure and displacement data were prepared in the same manner as described in Figure 3. The amplitudes are scaled for illustration but the relative amplitudes for the two displacements (2nd and 3rd traces) remain preserved. (a) S005 for event 2007.335.01.44 (year.julian-day.hour.minute of the origin time) with focal depth of $41 \mathrm{~km}$ and epicentral distance of $34^{\circ}$. The records are $1000 \mathrm{~s}$ long band-pass filtered at $0.01-0.05 \mathrm{~Hz}$ showing $P$, $S$, and $S S$ arrivals. (b) EOS1 for an $M_{\mathrm{w}} 7.3$ event 2012.312.16.35 near Guatemala with depth $24 \mathrm{~km}$ and epicentral distance $129.3^{\circ}$. The $600 \mathrm{~s}$ section filtered at $0.02-0.1 \mathrm{~Hz}$ contains $P_{\text {diff }}, P K I K P$, and $P P$. These phases are either ambiguous or unidentifiable on original seismograms except for the large amplitude $P P$.

records. A record section comprising corrected EOS1 and the neighboring land station waveforms for a remote Guatemala magnitude 7.5 earthquake exhibits consistency throughout the section (Fig. 7).

The CWB real-time ocean-bottom observatory has a mission to enhance the performance of early warning for offshore megaquakes and their potential for tsunamis. Accurate knowledge of offshore seismogenic structures is therefore critical to this mission. Local events received by EOS1 are usually shrouded in the infragravity-wave-induced noise. In this study, we analyze 13 events for EOS1 during 2012 (Fig. 8). These events are among those that were issued by CWB as island-wide-felt events, usually with $M_{\mathrm{L}}>3.5$, which triggered the Institute of Earth Sciences (IES) semiautomatic quick-centroid moment tensor (CMT) procedure (Kao et al., 1998). They were selected for locations either offshore or between EOS1 and the majority of Broadband Array in Taiwan for Seismology (BATS). One-hour data

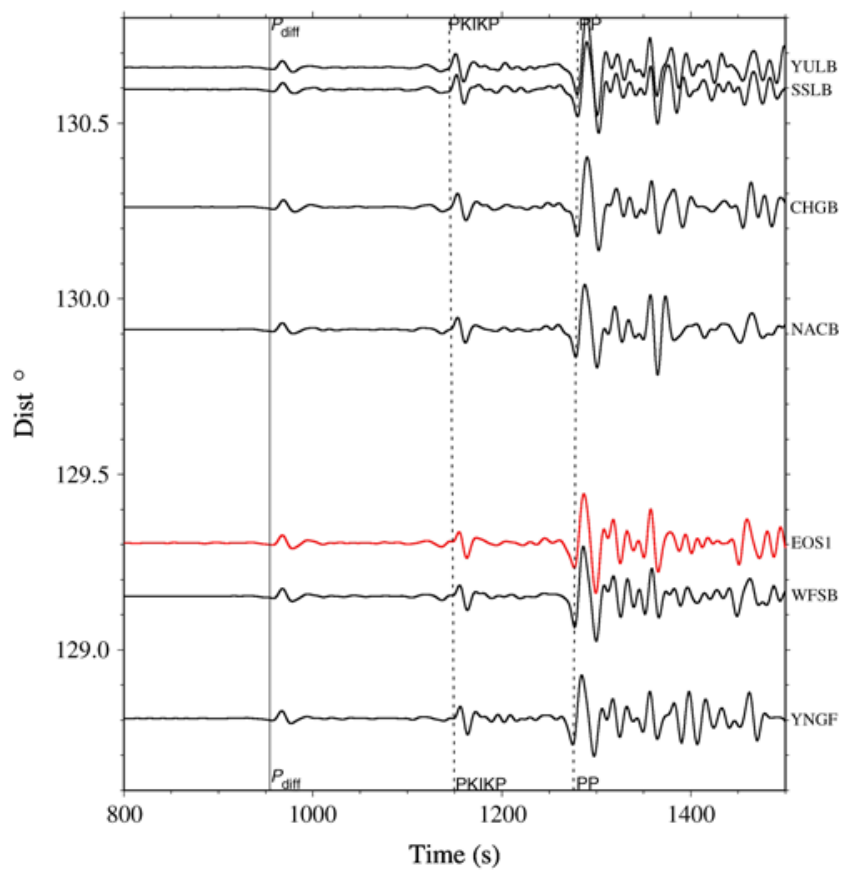

Figure 7. A displacement waveform section for the $M_{\mathrm{w}} 7.3$ Guatemala event in Figure 6b composed of EOS1 and BATS and the F-net (YNGF) stations, all filtered at $0.02-0.1 \mathrm{~Hz}$. The corrected EOS1 waveforms are highly consistent with those on the neighboring land stations. The relative geometry of the stations is shown in Figure 8. The color version of this figure is available only in the electronic edition.

windows were prepared for these local events by cutting the window at $1000 \mathrm{~s}$ before the event origin time.

Examples of seismograms of EOS1 for local events with $M_{\mathrm{L}} 4.3$ and 3.8 are shown in Figure 9. The $P$ wave is a slight disturbance imbedded in the displacement record at a travel time of about $10 \mathrm{~s}$, which otherwise correlates with the pressure almost wiggle by wiggle. Application of the transfer function cleans the seismograms and reveals a prominent $P$ arrival starting at the expected arrival time. The magnitudes of the other 10 events range from $M_{\mathrm{L}} 3.6$ to 5.3, and only 2 events with $M_{\mathrm{L}} 5.1$ and 5.3 (11 and 12, respectively, on Fig. 8) have large enough $P$-wave signal-to-noise ratios that wave noise removal is unnecessary.

The overall effect of removing the wave loading noise from the vertical velocity is to remove the noise hump caused by infragravity waves in the spectrum, and this can be best seen in the EOS1 data because it records the most energetic infragravity waves among all the OBSs analyzed in this study. We extended the one-hour transfer function to one-day transfer function and applied the noise removal to each day's velocity record from EOS1, and calculated the PSD for the cleaned data in the same manner as for the original data using the McNamara and Buland (2004) routine (Fig. 2). The noise hump from 0.1 to below $0.01 \mathrm{~Hz}$ on the EOS1 vertical spectrum is removed and the maximum reduction in noise level reaches $25-30 \mathrm{~dB}$. This amount of reduction is comparable 


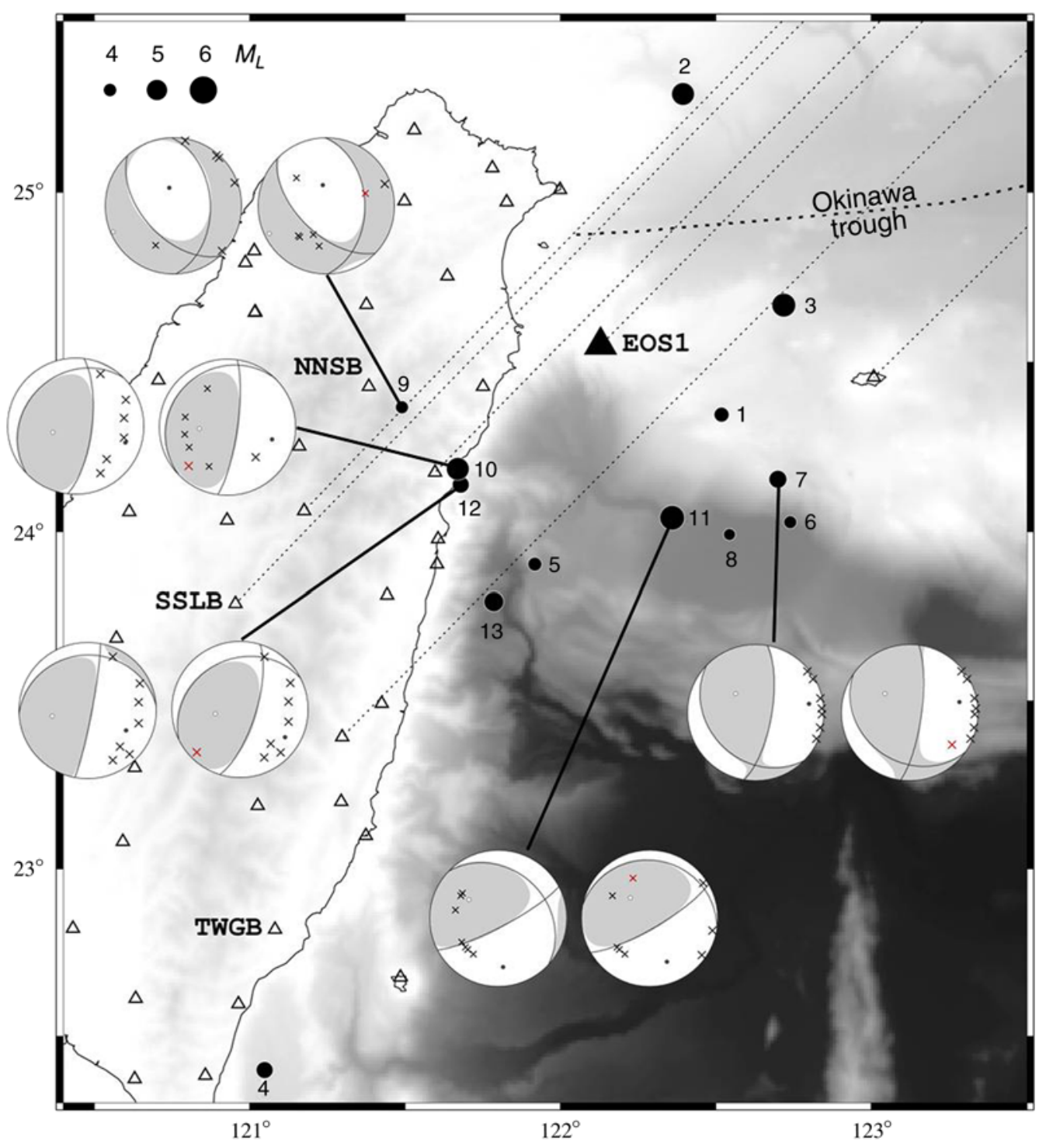

Figure 8. Thirteen local events for which the EOS1 seismograms have been corrected using the transfer function and the centroid moment tensor's (CMT) determined. Symbol size is proportional to $M_{\mathrm{L}}$ (see legend on the upper left). CMT solutions without (left) and with (right) EOS1 for events 7, 9, 10, 11, and 12 are shown as examples. Stations are projected to lower hemisphere as crosses. Open triangles are BATS stations and YNGF. The three BATS stations labeled are those for the additional inversion for event 7 shown in Figure 10. Also shown here are the great circle paths (thin dashed line) for the Guatemala event received by stations surrounding EOS1 (see Fig. 7). The color version of this figure is available only in the electronic edition.

to that for a selected one-hour data window from MOBB (Dolenc et al., 2007).

\section{CMT Solution with EOS1}

After CWB reports an island-wide-felt earthquake with epicenter and magnitude $M_{\mathrm{L}}$ determinations, the IES data management center launches a quick-CMT inversion using three combinations of stations: (1) those with the best azimuthal coverage; (2) best signal-to-noise ratio; and (3) shortest average distance (Kao et al., 1998), and reports the solution that has the smallest average misfit for all stations among the three schemes. This procedure takes a maximum of seven BATS stations with a minimum of three stations. The epicen- ter is fixed at the CWB location but the depth and magnitude are allowed to vary. The inversion employs a simple velocity model composed of two crustal layers on top of a mantle. In this study, the Moho discontinuity is set at 40 and $25 \mathrm{~km}$ for BATS and EOS1, respectively, with the crustal and mantle velocities fixed for all stations. The quick-CMT procedure explores a variety of bandwidths, but the bandwidth is set at $0.03-0.08 \mathrm{~Hz}$ for the results described here.

In Figure 8, we show CMT solutions for five events each of which has a misfit for EOS1 comparable to that for BATS stations. The other eight events have relatively poor fit at EOS1 resulting probably from the complex structure at the junction of the subduction zone and the Eurasian lithosphere. Two of the five events reported in this study achieved 
(a)

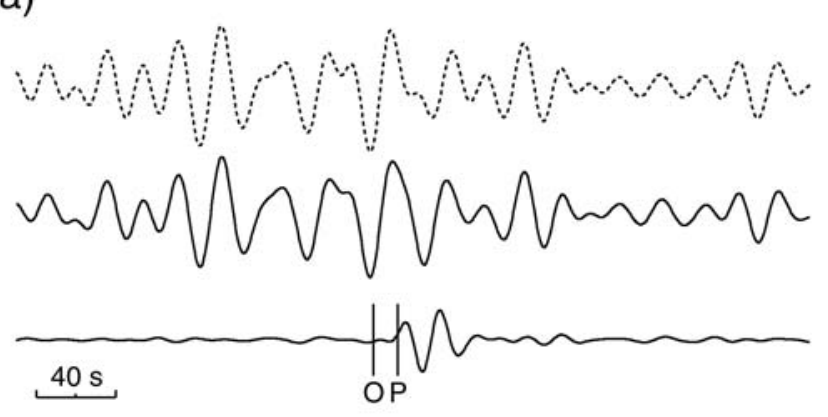

(b)

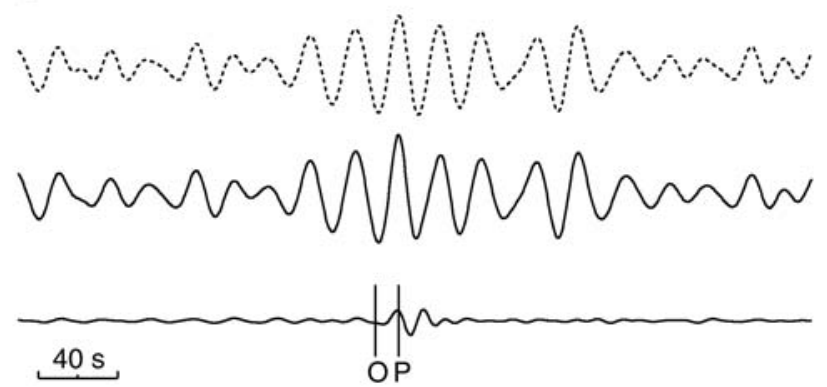

Figure 9. Example of noise removal from EOS1 vertical component for two local events. (a) Event 7, 2012.064.17.52, depth $46 \mathrm{~km}, M_{\mathrm{L}} 4.3$, band-pass filtered at $0.03-0.08 \mathrm{~Hz}$. (b) Event 9, 2012.084.15.03, depth $6 \mathrm{~km}, M_{\mathrm{L}} 3.75$, band-pass filtered at 0.04-0.1 Hz. See Figure 8 for locations. Plotting style is the same as for Figure 6. $P$ waveforms are completely buried in noise on the original records but prominent after correction. Vertical bars mark the origin time $(O)$ and the arrival time of $P$ wave $(P)$.

reduced average misfit when the waveform from EOS1 was included in the inversion. Because the inversion is predominantly controlled by BATS data, adding a vertical component of EOS1 has overall a small influence on the CMT solution. A general similarity in CMT solutions was also observed for offshore California events when data from the Monterey ocean-bottom broadband seismic station (MOBB) was incorporated with land data (Taira et al., 2013). However, both MOBB and EOS1 can provide critical constraints that yield more reliable solutions for some events than land stations alone. Events 9 and 12 are examples of such cases with EOS1 at critical position although results may not differ significantly in this study (Fig. 8). More data and better velocity models are required to verify whether or not the differences in CMT with and without EOS1 are sufficiently systematic to warrant further interpretation.

To illustrate the influence of the offshore constraint, we manually picked only three BATS stations and ran the inversion for event 7 . Figure 10 shows a typical output of the quick-CMT inversion routine at IES. The fitting of the waveforms is overall good and the average misfit is reduced with the additional constraint from EOS1 although the CMTs remain largely similar. The predicted $P$ wave is delayed at EOS1 even though the Moho is set at $25 \mathrm{~km}$ for this site as opposed to $40 \mathrm{~km}$ for BATS stations. This event illustrates the possibility of further improvements in CMT estimation for these earthquakes through considering the ocean-continent transitional nature of this region.

\section{Discussion}

The transfer function reflects the instrument properties and the seafloor structure, and not the ocean conditions (Crawford et al., 1991). All of the portable broadband OBSs use the same type of seismic sensor, that is, Güralp CMG3TC, and the same type of DPGs. The seismometer is usually well calibrated compared with DPGs. Taira et al. (2013) documented a systematic $20 \%$ drop in the amplitude of the transfer function at $75 \mathrm{~s}$ for MOBB after the DPG was replaced by nominally the same type of instrument. This prohibits a first-order interpretation of the relative amplitude of transfer function between OBSs at different sites. However, this problem may be partially alleviated in this study. The same OBS with the same pairing of seismometer and DPG was deployed at S002 and S005 in consecutive years, and again for S0904 and S1001 (see Table 1). The relative amplitudes of the transfer functions between S002 and S005 and between S0904 and S1001 shown in Figure 5 thus should be related to differences in sediment and/or crustal structure.

Another complicating factor is that water depth controls the wavelength of the infragravity waves at a site for a given frequency and thus the transfer (compliance) function depends on water depth. This bias can be largely neutralized by multiplying the transfer function by the wavenumber $k$ of the infragravity wave determined from the dispersion relation for an ocean gravity wave

$$
\omega^{2}=g k \times \tanh (k H),
$$

in which $\omega$ is the angular frequency, $g$ is gravitational acceleration, and $H$ is water depth. Figure 5 h shows the transfer function times $k$ versus $k$. The wavenumber-normalized transfer functions for S005 and S1001 have largest amplitudes among the five, and the amplitudes decrease for S002 and S0904. S004 has a transfer function concentrated in low frequencies and is difficult to compare it with the amplitudes for other OBSs.

To test the correlation between transfer function and the shallow crustal structure, we estimated the sediment thickness from published seismic-reflection profiles traversing as closely to each OBS site as possible (Schnürle et al., 1998; Hsu, 2013; Table 1). The sediment thickness is estimated to be $700 \mathrm{~m}$ for $\mathrm{S} 002$ and $567 \mathrm{~m}$ for S005. Both the thickness and the degree of compaction of the sediment control the amplitude of the $k$-normalized transfer function. Because the thicknesses do not differ substantially, whether the relatively high compliance at S005 is due to the low shear strength in the sediment and/or in the shallow crust is difficult to infer. The sediments are 276 and 935 m thick beneath S0904 and S1001, respectively, and the amplitude of the normalized transfer function for S1001 is higher than that for S0904. 
(a) Event ID: 201203041752
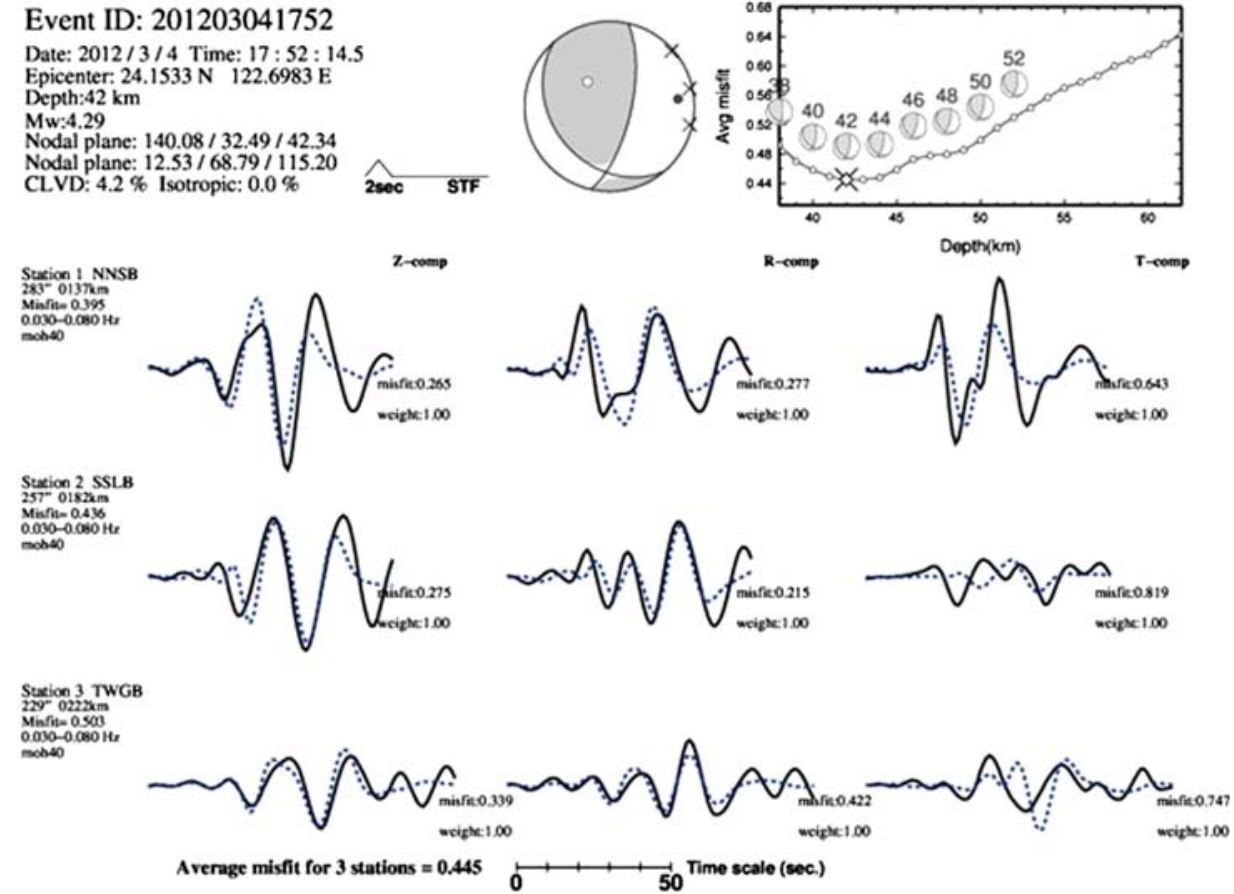

(b)

Event ID: 201203041752

Date: $2012 / 3 / 4$ Time: $17: 52: 14.5$ Epicenter: $24.1533 \mathrm{~N} \quad 122.6983 \mathrm{E}$ Depth:47 km Mw: 4.30 Nodal plane: $116.62 / 30.22 / 20.64$ Nodal plane: $8.59 / 79.78 / 118.59$ CLVD: $31.5 \%$ Isotropic: $0.0 \%$
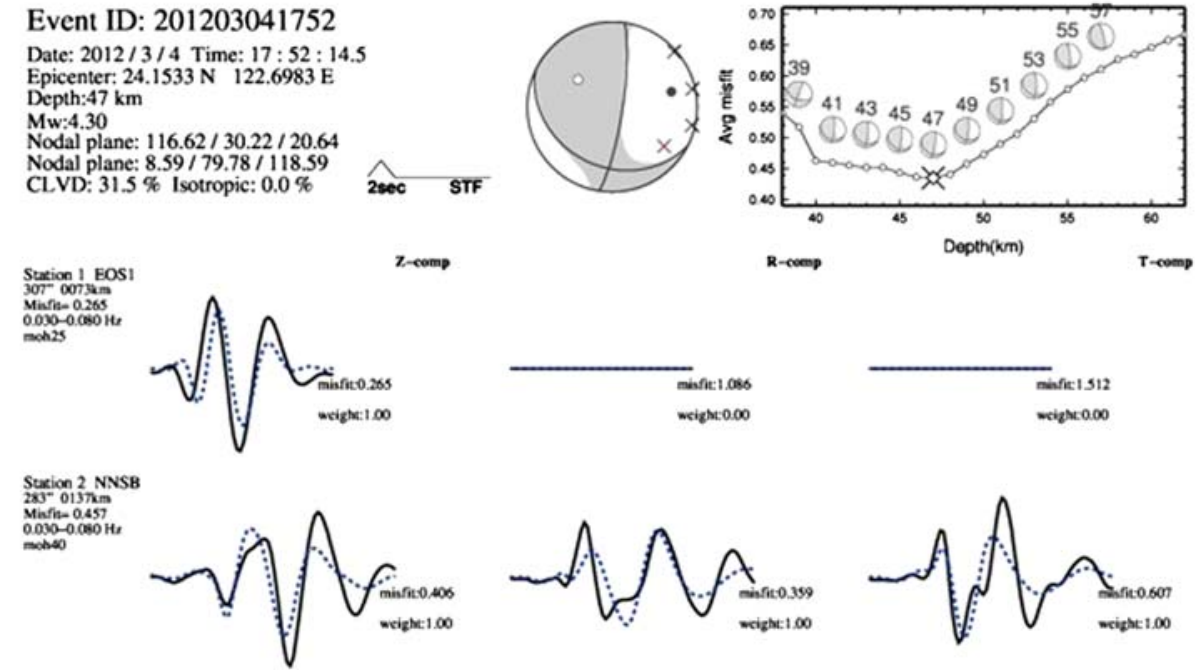

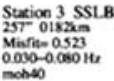
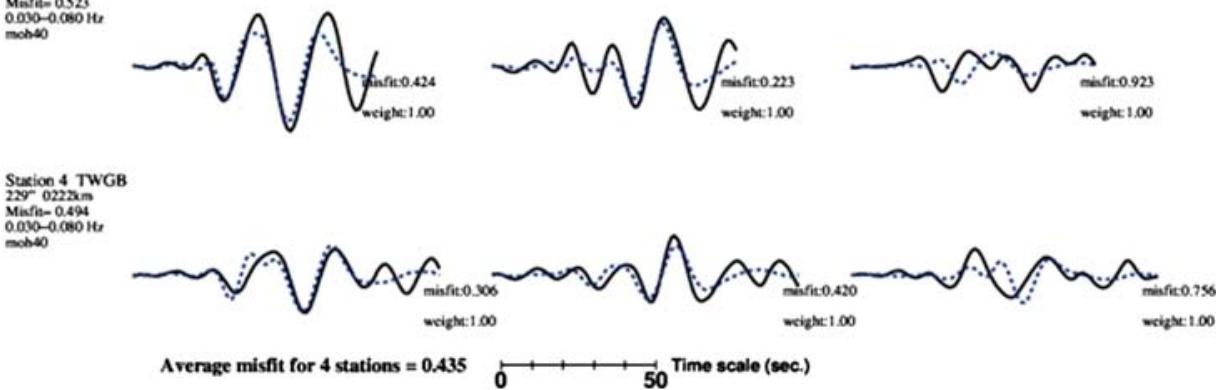

Figure 10. Output of Institute of Earth Sciences quick-CMT inversion for event 7, 2012.064.17.52 (March 4), (a) without and (b) with EOS1. In (a) the land stations are NNSB, SSLB, and TWGB from top to bottom rows; the left, middle, and right columns in each row are vertical, north, and east components of displacement, respectively. In (b), display is the same except the first row is for EOS1. Horizontal data of EOS1 are not considered in the inversion. Observed and calculated waveforms are in solid line and dotted line, respectively. Band-pass filter is $0.03-0.08 \mathrm{~Hz}$. With EOS1, the average misfit is decreased from 0.445 to 0.435 and the event depth is slightly increased from 42 to $47 \mathrm{~km}$. The CMT is not exactly the same as that shown in Figure 8 because the station numbers are reduced in this test. The color version of this figure is available only in the electronic edition. 
The S0904 site is at the western slope of the Luzon arc where piling up of sediment may be inefficient, whereas S1001 was deployed at the abyss of the Huatung basin adjacent to the Luzon arc, where supply of sediment from the high-elevation arc may be abundant. The significant discrepancy in sediment thickness between S0904 and S1001, that is, 276 versus $935 \mathrm{~m}$, probably contributes to the relative amplitude of the normalized transfer function between them. An inverse modeling will be conducted in the future to quantify the sediment/crustal effects on the compliance functions observed at these OBSs.

In this study, we did not address the noise that dominates the horizontal seismic data of OBSs. Lin et al. (2010) show much higher noise level on the horizontals than on the vertical for the pop-up OBSs, indicating both low shear velocity of the sediment and poor coupling to the seafloor allowing tilting under seafloor current. We found moderately higher horizontal noise compared to the vertical for the shallowburied EOS1 (not shown in this study). Crawford and Webb (2000) applied the transfer function technique to remove the tilt effect of an OBS. Dolenc et al. (2007) reported that this tilt effect is small for MOBB due to a good installation. Our preliminary work verifies that EOS1 sensor is leveled to an accuracy for which noise from tilt is hard to discern.

\section{Conclusions}

The transfer functions and coherences established in this study accurately describe how much of the bottom motion is forced by wave loading as seen in the pressure record. The application of the transfer function successfully removed most, if not all, of the noise originating from infragravity waves for frequencies from up to 0.05 to below $0.01 \mathrm{~Hz}$. This operation helps isolate from background noise seismic phases that are central to seismology, and the effect is especially evident for the cabled OBS installed on the shallow seafloor. We inferred that, when the difference in sediment thickness is significant, the relative amplitude of the wavenumber-normalized transfer function between two OBS sites may be dominated by the sediment thickness variation. The next step after construction of the transfer function is to invert for the elastic properties of the sediment and shallow crust after the instrument responses of both the seismometer and the DPG are accurately accounted for. This requires an accurate calibration of the DPGs. In addition, a near-real-time approach of noise removal by Taira et al. (2013) may be a future component of the EOS1 analyses for a better quickCMT inversion.

\section{Data and Resources}

The ocean-bottom seismograph (OBS) data used in this study are maintained by the OBS lab of Institute of Earth Sciences (IES) and are shared on a collaboration basis. The Broadband Array in Taiwan for Seismology (BATS) data are maintained by IES and can be accessed at http://tecdc .earth.sinica.edu.tw (last accessed January 2013). Plots in this paper were made using Generic Mapping Tools version 4.2.1 (www.soest.hawaii.edu/gmt; last accessed January 2013; Wessel and Smith, 1998). Waveform data for YNGF were extracted from F-net at http://www.fnet.bosai.go.jp (last accessed August 2013). The McNamara and Buland (2004) power spectrum density (PSD) analysis tools were downloaded from http://geohazards.cr.usgs.gov/staffweb/ mcnamara/Software/PDFSA.html (last accessed January 2013). The code "sacpsd" was downloaded from http://www .eas.slu.edu/eqc/eqc_cps/SACPSD (last accessed September 2013).

\section{Acknowledgments}

This research was supported by National Science Council, Taiwan, under Grants NSC 101-2811-M-001-091 and NSC 102-2627-E-001-001. We appreciate the careful and constructive comments from the associate editor and an anonymous reviewer. We thank the captain and crew of R/V Ocean Researcher 1 and the staff of the Institute of Oceanography, National Taiwan University, for their support of the OBS campaigns since 2006.

\section{References}

Cox, C. S., T. Deaton, and S. C. Webb (1984). A deep sea differential pressure gauge, J. Atmos. Ocean. Tech. 1, 237-246.

Crawford, W. C., and S. C. Webb (2000). Identifying and removing tilt noise from low-frequency $(<0.1 \mathrm{~Hz})$ seafloor vertical seismic data, Bull. Seismol. Soc. Am. 90, 952-963.

Crawford, W. C., S. C. Webb, and J. A. Hildebrand (1991). Seafloor compliance observed by long-period pressure and displacement measurements, J. Geophys. Res. 96, 16,151-16,160.

Crawford, W. C., S. C. Webb, and J. A. Hildebrand (1999). Constraints on melt in the lower crust and Moho at the East Pacific Rise, $9^{\circ} 48^{\prime} \mathrm{N}$, using seafloor compliance measurements, J. Geophys. Res. 105, 2923-2939.

Dolenc, D., B. Romanowicz, P. McGill, and W. Wilcock (2008). Observations of infragravity waves at the ocean-bottom broadband seismic stations Endeavour (KEBB) and Explorer (KXBB), Geochem. Geophys. Geosys. 9, doi: 10.1029/2008GC001942.

Dolenc, D., B. Romanowicz, D. Stakes, P. McGill, and D. Neuhauser (2005). Observations of infragravity waves at the Monterey ocean bottom broadband station (MOBB), Geochem. Geophys. Geosys. 6, doi: 10.1029/2005GC000988.

Dolenc, D., B. Romanowicz, R. Uhrhammer, P. McGill, D. Neuhauser, and D. Stakes (2007). Identifying and removing noise from the Monterey ocean bottom broadband seismic station (MOBB) data, Geochem. Geophys. Geosys. 8, doi: 10.1029/2006GC001403.

Hsu, H.-H. (2013). Seismic imaging of sediment dispersal systems: Southwest and northeast Taiwan Perspectives, Ph.D. thesis, National Taiwan University, 166 pp. (in Chinese).

Kao, H., P. Jian, K. Ma, B. Huang, and C. Liu (1998). Moment-tensor inversion for offshore earthquakes east of Taiwan and their implications to regional collision, Geophys. Res. Lett. 25, 3619-3622.

Ko, Y. T., B. Y. Kuo, and S. H. Hung (2012). The southwestern edge of the Ryukyu subduction zone: A high $Q$ mantle wedge, Earth Sci. Planet. Lett. 335/336, 145-153, doi: 10.1016/j.epsl.2012.04.041.

Kuo, B. Y., W. C. Chi, C. R. Lin, E. T. Chang, J. Collins, and C. S. Liu (2009). Two-station measurement of Rayleigh-wave phase velocities for the Huatung basin, the westernmost Philippines Sea, with OBS: Implications for regional tectonics, Geophys. J. Int. 179, 18591869, doi: 10.1111/j.1365-246X.2009.04391.x.

Kuo, B. Y., C. C. Wang, S. C. Lin, C. R. Lin, P. C. Chen, J. P. Jang, and H. K. Chang (2012). Shear-wave splitting at the edge of the Ryukyu subduc- 
tion zone, Earth Planet. Sci. Lett. 355/356, 262-270, doi: 10.1016/ j.eps1.2012.08.005.

Lin, C. R., B. Y. Kuo, W. T. Liang, W. C. Chi, Y. C. Huang, J. Collins, and C. Wang (2010). Ambient noise and teleseismic signals recorded by ocean-bottom seismometers offshore eastern Taiwan, Terr. Atmos. Ocean. Sci. 21, 743-755, doi: 10.3319/TAO.2009.09.14.01(T).

McNamara, E. E., and R. P. Buland (2004). Ambient noise levels in the continental United States, Bull. Seismol. Soc. Am. 94, 1517-1527, doi: $10.1785 / 012003001$.

Peterson, J. (1993). Observations and modeling of seismic background noise, U. S. Geol. Surv. Tech. Rept. 93-322, 95 pp.

Schnürle, P., C. S. Liu, S. Lallemand, and D. Reed (1998). Structural controls of the Taitung canyon in the Huatung basin east of Taiwan, Terr. Atmos. Ocean. Sci. 9, 453-472.

Taira, T., Z. Zheng, and B. Romanowicz (2013). On the systematic long period noise reduction on Ocean floor broadband seismic sensors collocated with differential pressure gauges, Bull. Seismol. Soc. Am. 104, 247-259, doi: 10.1785/0120130015.

Webb, S. C. (1998). Broadband seismology and noise under the ocean, Rev. Geophys. 36, 105-142.

Webb, S. C., and W. C. Crawford (1999). Long-period seafloor seismology and deformation under ocean waves, Bull. Seismol. Soc. Am. 89, $1535-1542$

Webb, S. C., X. Zhang, and W. Crawford (1991). Infragravity waves in the deep ocean, J. Geophys. Res. 96, 2723-2736.

Wessel, P., and W. H. F. Smith (1998). New, improved version of generic mapping tools released, Eos Trans. AGU 79, 579.
Institute of Earth Sciences

Academia Sinica

POB 1-55 Nangang

Taipei, Taiwan

byk@earth.sinica.edu.tw

crlin@earth.sinica.edu.tw

wtl@earth.sinica.edu.tw

(B.-Y.K., C.-R.L., W.-T.L.)

Lamont-Doherty Earth Observatory

61 Route 9W

Palisades, New York 10964

scw@1deo.columbia.edu

(S.C.W.)

Central Weather Bureau

64, Gongyuan Road

Taipei, Taiwan

naigi@cwb.gov.tw

(N.-C.H.)
Manuscript received 30 October 2013; Published Online 22 July 2014 